\title{
129 DEVELOPMENT OF AB-201, A NOVEL ALLOGENEIC ANTI-HER2-SPECIFIC CAR-NK CELL THERAPY FOR THE TREATMENT OF HER2+ TUMORS
}

${ }^{1}$ Hoyong Lim, ${ }^{2}$ Amanda Medcalf, ${ }^{2}$ Lisa Guerrettaz, ${ }^{1}$ Eun Ji Choi, ${ }^{1}$ Hansol Kim, ${ }^{1}$ Bitna Yang, ${ }^{1}$ Eun Ji Kim, ${ }^{1}$ Eun-Sol Lee, 'Jeong Min Kim, ${ }^{1}$ Yusun Kim, ${ }^{1}$ Bokyung Min, 'Sang-Min Paik, ${ }^{1}$ Hyeong Jin Nam, ${ }^{1}$ Seungryel Han, ${ }^{2}$ Srinivas Somanchi, ${ }^{2}$ Eugene Helsel, ${ }^{2} J a s o n$ Litten, ${ }^{2}$ Peter Flynn, ${ }^{2}$ Heather Raymon*, 'Yu-Kyeong Hwang. ${ }^{1} G C$ LabCell, Yongin, Republic of Korea; ${ }^{2}$ Artiva Biotherapeutics, San Diego, CA, USA

Background Human Epidermal Growth Factor Receptor 2 (HER2), is a receptor tyrosine kinase that is highly expressed on the surface of many solid tumors. While many patients derive meaningful benefit from the approved HER2-directed therapies, most will eventually suffer relapse or progression of their disease highlighting the need for additional treatment options. Currently there are no FDA-approved cellular therapies targeting HER2. Over the past decade, however, cellular therapy has been shown to be a viable treatment option in different cancer types. Here we present AB-201, an off-theshelf, cryopreserved cord blood (CB)-derived HER2 chimeric antigen receptor (CAR)-natural killer (NK) cell therapy as a safe, active, and readily available option for patients with HER2 + solid tumors.

Methods AB-201 is comprised of ex vivo expanded allogeneic CB-derived NK cells that have been genetically modified to express a HER2-directed CAR and presented as a cryopreserved infusion-ready product. The manufacturing process utilizes a feeder-cell line engineered to express factors specifically identified as supportive to NK cell expansion and a lentiviral transduction step to introduce the HER2 CAR construct. In vitro characterization of AB-201 included evaluation of the purity and expression of cell surface markers by flow cytometry and short- (4 hour) and long-term (over 5 days) cytotoxicity assays in the presence of HER2+ tumor cell lines at various effector to target ratios. In addition, AB-201 efficacy was assessed in vivo in established ovarian (intraperitoneal, SKOV-3), breast (intraperitoneal, HCC1954) and gastric (subcutaneous, N87) xenograft models in NSG mice.

Results HER2 CAR expression was detected in $93.1 \%$ of AB201 cells. AB-201 is 97.9\% CD3-/CD56+ cells and $94.6 \%$ CD56+/CD16+. Further characterization of AB-201 demonstrated high expression of $\mathrm{NK}$ activating receptors such as NKG2D, NKp30, NKp46, and DNAM-1 and expression of the chemokine receptor, CXCR3. AB-201 demonstrated concentration-dependent and HER2 targeted short-term cytotoxic activity and sustained long-term cell killing against the tumor cell lines SKOV-3, HCC1954, and NCI-N87. Efficacy, as evidenced by a significant reduction in bioluminescent signal or tumor volume, was observed in all xenograft models. A significant survival benefit over non-transduced NK cells or trastuzumab controls was demonstrated in the HCC1954 model.

Conclusions Data presented herein suggests that AB-201, a highly pure and readily expandable HER2-directed CAR NK cell product, has potential to be an effective therapy in the treatment of HER2+ tumors.

Ethics Approval The animal studies were conducted in accordance with an Institutional Animal Care and Use Committeeapproved protocol and with the approval of an IACUC committee at each center where the studies took place

http://dx.doi.org/10.1136/jitc-2021-SITC2021.129 